14

\title{
Аппаратно-программный комплекс анализа походки пациентов с нормотензивной гидроцефалией
}

\author{
(ㄷ Г.В. Гаврилов, ${ }^{1}$ С.Г. Ястребов, ${ }^{2,}$ Д.Б. Вчерашний, ${ }^{3,2}$ Д.В. Свистов ${ }^{1}$ \\ ${ }^{1}$ Военно-медицинская академия им. С.М. Кирова, \\ 194044 Санкт-Петербург, Россия \\ ${ }^{2}$ Физико-технический институт им. А.Ф. Иоффре РАН, \\ 194021 Санкт-Петербург, Россия \\ ${ }^{3}$ Санкт-Петербургский государственный университет, медицинский фракультет, \\ 199106 Санкт-Петербург, Россия \\ ฯ e-mail: yastrebov@mail.ioffe.ru
}

(Поступило в Редакцию 5 декабря 2017 г.)

\begin{abstract}
Предложен новый метод анализа походки пациентов с нормотензивной гидроцефалией, а также с рядом других патологических заболеваний, сопровождающихся нарушением походки, в частности, таких как болезни Паркинсона и Альцгеймера. С этой целью с помощью ультразвукового дальномера записывается грессограмма - зависимость расстояния, пройденного пациентом от времени и ее производная, которая анализируется с помощью преобразования Фурье. Выполненный анализ позволяет определить такие значимые параметры походки как общее время, затрачиваемое на прохождение дистанции, общее количество шагов, средняя длина шага, среднее, минимальное и максимальная значения маршевой скорости движения и среднее время, затрачиваемое на один шаг. Сравнение параметров походки до и после тап-теста делает возможным оценить перспективность проведения операции вентрикулоперитонеального шунтирования у пациента с нормотензивной гидроцефалией, а их определение после операции позволяет сделать вывод о ее эффективности. В случае же болезни Паркинсона положительная динамика при оценке походки описанным методом на фоне приема леводопы позволит провести дифференциальную диагностику данного заболевания с нормотензивной гидроцефалией. В работе показано, что к состоянию больного нормотензивной гидроцефалией чувствительны такие параметры как - среднее значение скорости, амплитуда разброса мгновенных скоростей на маршевой дистанции, время, затрачиваемое на один шаг и, как следствие, средняя длина шага. Важным инструментом анализа, как оказалось, представляются ритмограммы, позволяющие выявить нарушения ритма походки. Разработанный прибор в сочетании с набором алгоритмов могут быть использованы при создании аппаратно-программного комплекса сбора телеметрической информации для экспресс-анализа походки.
\end{abstract}

DOI: 10.21883/JTF.2018.10.46506.2587

\section{Введение}

Своевременная диагностика неврологических заболеваний важна для решения задач профилактики и оценки эффективности лечения неврологических заболеваний, в ряду которых находится нормотензивная гидроцефалия. В настоящее время одним из эффективных инструментов диагностики степени тяжести этой патологии является анализ походки пациента, который проводится либо визуально, либо с использованием дорогостоящего оборудования. Более того, в настоящее время нет работ, исследующих чувствительность параметров походки к влиянию на них медицинских манипуляций, таких как люмбальная пункция и операция по установлению шунта, отводящего ликвор. Целью настоящей работы является применение физического метода сбора телеметрической информации о походке пациентов, пройденного пациентом пути в функции времени, с помощью дешевого и безопасного ультразвукового дальномера, а также разработка научных основ ее анализа.

Известно, что при гидроцефалии происходит излишнее скопление ликвора в желудочках головного мозга и в субарахноидальных пространствах, что часто сопровождается увеличением внутричерепного давления, за исключением случаев нормотензивной гидроцефалии, когда давление ликвора не превосходит нормальных показателей. По-видимому, эффект повреждающего воздействия на мозг ликвора при нормальном давлении, связан с потерей эластических свойств мозга, а также изменением его проницаемости для ликвора [1]. Поэтому происходит расширение внутрижелудочкового пространства и деформация тканей мозга. Эта патология сопровождается тремя симптомами, так называемой классической триадой Хаким-Адамса, основной составляющей которой является нарушение походки [2]. Частота передвижения ног пациента значительно отличается от ее среднего значения из-за их „подволакивания“ и спонтанных кратковременных остановок на дистанции. По сравнению с возрастной нормой увеличивается среднее время, затрачиваемое на один шаг, длина шага уменьшается, ухудшаются также скоростные характеристики. Пациент жалуется на неуверенность и замедление ходьбы. Отметим, что нарушение походки наступает на ранних стадиях болезни, а при своевременной диагностике и 

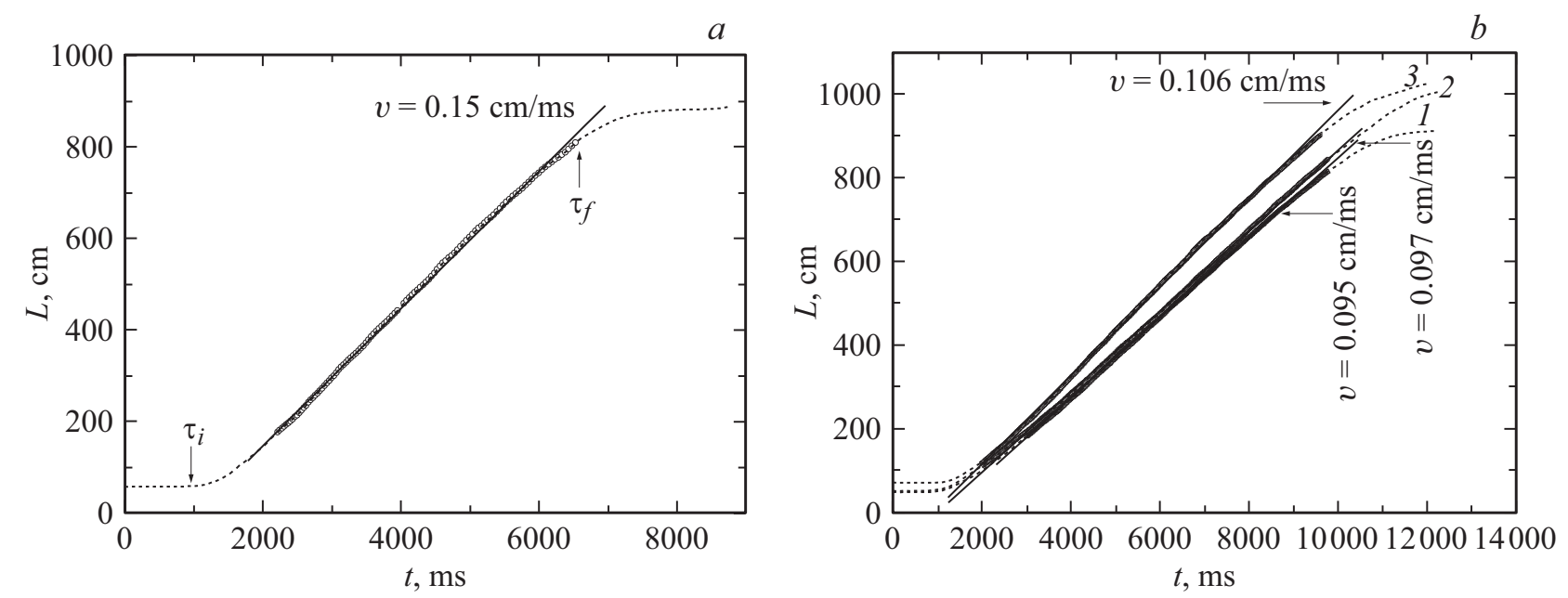

Рис. 1. Грессограммы. Пунктирной кривой показана зависимость пройденного пути от времени, символами выделен линейный участок на зависимости, греческими буквами указаны времена начала и окончания движения соответственно. Прямые линии проведены методом наименьших квадратов через точки линейного тренда (соответствует движению на маршевом участке пути). Для маршевого движения приведены значения средних скоростей движения. $a-$ грессограмма здорового человека; $b-$ грессограммы пациента с нормотензивной гидроцефалией: 1 - до тап-теста, 2 - после тап-теста, 3 - после операции.

имплантации шунта развитие патологического процесса можно приостановить, не дожидаясь проявления других симптомов. Если не оказать пациенту своевременной хирургической помощи, то его, в конечном счете, ждет тяжелая инвалидизация.

Известно, что для диагностики нормотензивной гидроцефалии применяется так называемый тап-тест, при котором пациенту проводят люмбальную пункцию, выводят достаточно большое количество ликвора $(30-60 \mathrm{ml})$, в результате которой снижается давление ликвора на мозг и состояние пациента улучшается, что, в частности, проявляется в регрессе нарушений походки (последняя становится ритмичнее, уменьшается время прохождения дистанции за счет увеличения скорости) [3-8]. Напомним, что для дифференциального диагноза с болезнью Паркинсона используется нагрузка пациента леводопой $[9,10]$, в результате которой улучшаются отмеченные параметры походки. На основании положительного влияния тап-теста на походку делается заключение о перспективности проведения операции вентрикулоперитонеального шунтирования, поскольку такая операция, проведенная на ранней стадии заболевания, нормализует давление ликвора на мозг и исключает прогрессирование заболевания. Поэтому ранняя диагностика нормотензивной гидроцефалии весьма важна для сохранения здоровья и улучшения качества жизни пациентов и сохранности трудовых ресурсов. В том числе весьма важной представляется дифференциальная диагностика с болезнью Паркинсона [10].

Более того, контроль параметров походки также необходим для сопровождения пациента в послеоперационный период и для тонкой настройки параметров работы внешнерегулируемого клапана шунтирующей системы.

\section{Эксперимент, обсуждение и выводы}

Дальномер оригинальной конструкции состоит из источника зондирующего излучения, который испускает короткий ультразвуковой импульс. Импульс отражается от объекта и принимается сенсором. Рабочая частота $40 \mathrm{kHz}$. Расстояние рассчитывается, исходя из времени от испускания импульса до получения эха. Связь дальномера с ноутбуком осуществлялась с помощью контроллера. Телеметрические данные в виде зависимости пройденного от времени расстояния фиксировались и записывались на жесткий диск ноутбука.

Клинический пример. Пациент С., 75 лет в феврале 2017 г. находился на обследовании и лечении в клинике нейрохирургии Военно-медицинской академии им. С.М. Кирова. Больной предъявлял жалобы на шаткость походки и ухудшение памяти, мышления. Из анамнеза известно, что данная симптоматика развивалась в течение года и дебютировала с нарушения походки. В последующем присоединились когнитивные расстройства. Диагностика включала неврологический осмотр, МРТ головного мозга, допплерографию сосудов головы и шеи. Выполнена люмбальная пункция (давление ликвора составило $150 \mathrm{~mm} \mathrm{H}_{2} \mathrm{O}$ ) с дальнейшим проведением тап-теста, результат которого, как будет показано ниже, был положительным.

Телеметрическая информация с помощью дальномера собиралась у пациента С. до операции, при выполнении тап-теста, после операции, а также в сравнении с относительно здоровым человеком такого же возраста. Для оценки походки фиксировалось время работы дальномера и дистанция, пройденные больным нормотензивной гидроцефалией (пациент С.) и здоровым взрослым человеком. 

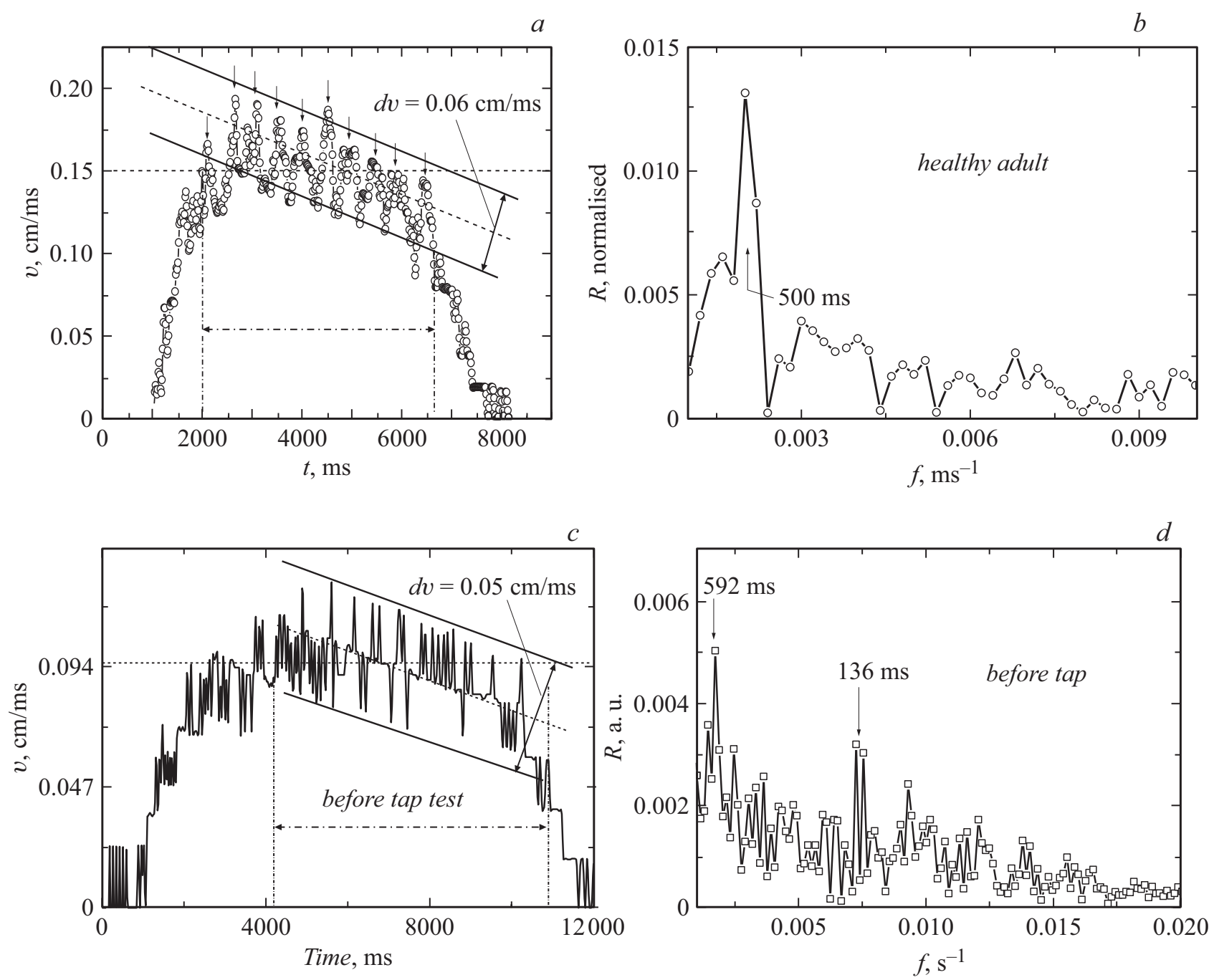

Рис. 2. $a, c, e, g$ - целеритограммы. Отрезками сплошных прямых выделены границы диапазона, в пределах которого осциллирует скорость. Стрелками показаны максимальные значения скорости, достигаемые на длине шага. Прерывистыми линиями показаны средняя скорость движения и амплитуда размаха осциллирующей части. Штрихпунктиром выделен участок, к которому применялось быстрое фурье-преобразование для построения периодограммы. $b, d, f, h-$ периодограммы поля скоростей. Стрелками выделены наиболее часто встречающиеся времена, затрачиваемые на перемещение пациента на один шаг: $a$ - целеритограмма здорового человека; $b$ - периодограмма мгновенных скоростей здорового человека; $c$ - целеритограмма пациента до тап-теста; $d$ - периодограмма скоростей пациента до тап-теста; $e$ - целеритограмма пациента до тап-теста; $f$ - периодограмма скоростей пациента после тап-теста; $g$ - целеритограмма пациента до операции; $h$ - периодограмма скоростей пациента после операции.

Для снятия телеметрической информации пациенту предлагалось наиболее комфортным для него способом пройти по прямой линии расстояние порядка $10 \mathrm{~m}$, при этом пройденное расстояние в функции времени движения измерялось ультразвуковым дальномером и записывалось в файл на жесткий диск. Для сравнения эту же дистанцию проходил здоровый человек мужского пола, рост которого приблизительно соответствовал росту пациента из-за известной связи средней длины шага и роста человека.

Зависимость пройденного пути от времени (,грессограмма“ от латинского слова „gressus“, что означает „шаг, ходьба“ ) для здорового человека, представлена пунктирной линией на рис. $1, a$, для пациента С. пред- ставлена прерывистой линией на рис. $1, b$. Стрелками на грессограмме, показаны характерные времена, при которых начинается и заканчивается движение: $\square_{i}$ и $\square_{f}$ соответственно. Участок на грессограммах, на котором происходит линейный рост пройденного расстояния от времени, можно назвать маршевым. Эти участки выделены на рисунках символами. Через участки выделения методом наименьших квадратов проведены отрезки линейной функции. Напомним, что линейная зависимость пройденного расстояния от времени характерна для случая движения с постоянной скоростью (в нашем случае имеет место постоянная средняя скорость). Производная от линейной зависимости в этом случае характеризует среднюю скорость движения и приведена на рисунках. 

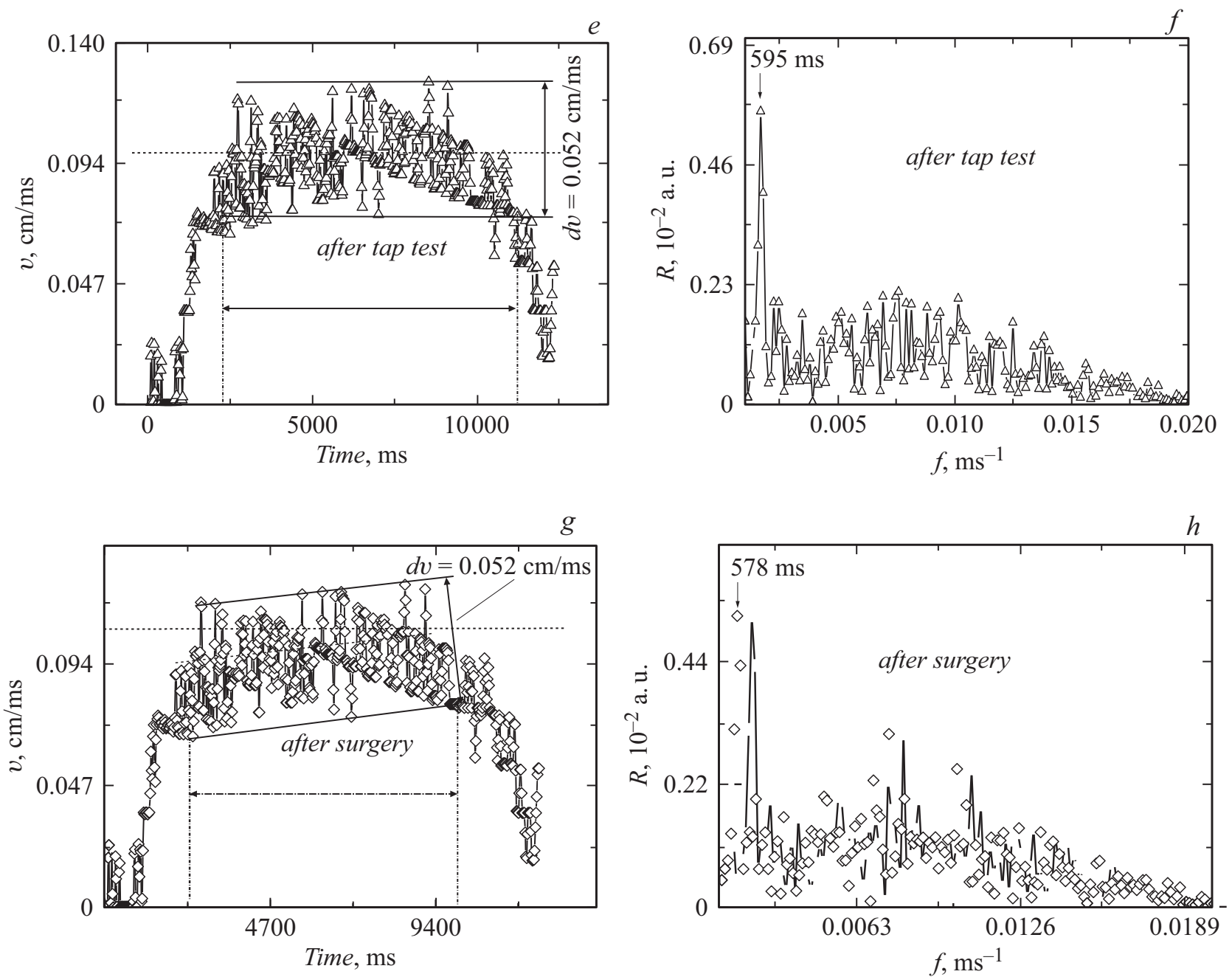

Рис. 2 (продолжение).

Абсолютная величина погрешности метода во всех рассмотренных случаях не превышала $10^{-4} \mathrm{~cm} / \mathrm{ms}$, поэтому на рисунках не приводится. Из рисунков видно, что максимальная скорость движения достигнута здоровым человеком, а минимальная - пациентом, над которым не проводились медицинские манипуляции. После проведения тап-теста и операции шунтирования средняя скорость движения пациента С. возросла.

Для более детального исследования грессограмм, исследовалась их производная по времени, дающая возможность исследовать отклонение мгновенной скорости движения пациента от ее среднего значения. Такие зависимости можно назвать целеритограммами, от латинского слова - „celeritas“, что означает „скорость“. Они приведены на рис. 2, a, c,e,g для здорового человека и пациента С., подвергнутого медицинским манипуляциям и до них. Видно, что в случае здорового человека на участке марша целеритограммы представляют собой осциллирующую периодическую функцию. Число максимумов на целеритограмме соответствует количеству шагов на марше. Целеритограммы пациента С. хаотичны, один период в движении выделить сложно. Для наглядности на целеритограммах отрезками прямых выделен диапазон изменения скорости при движении и отмечено значение амплитуды изменения скорости на марше, рассчитанное как разность между максимальным и минимальным значениями скорости.

Для более детального анализа к экспериментальным данным, приходящимся на маршевый участок, применялся алгоритм быстрого фурье-преобразования. Амплитуда этого преобразования, построенная в функции частоты, представляет собой периодограмму и представлена на рис. $2, b, d, f, h$. Над наиболее интенсивными пиками поставлены стрелки и приведены значения времени, затрачиваемого на один шаг, равному обратной величине частоты, над которой стоит стрелка.

На периодограмме здорового пациента выделяется одно значение периода, что свидетельствует о ритмичности его походки.

Из рисунков видно, что для пациента, не подвергавшегося медицинским манипуляциям, на периодограмме четко выделяются два периода, что свидетельствует об 

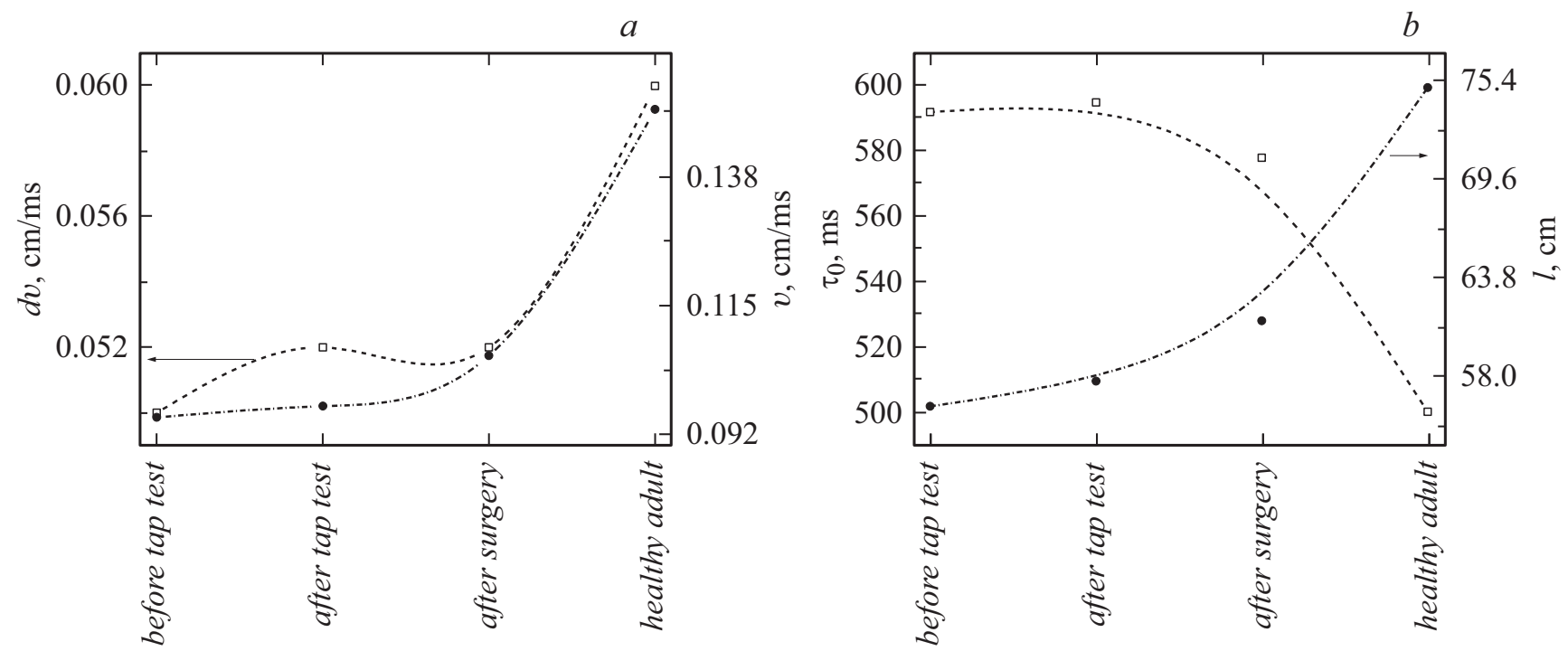

Рис. 3. $a-$ незаполненные квадраты - зависимость амплитуды колебаний мгновенной скорости от состояния пациента; кружки - средняя скорость движения из рис. $1 . b$ - прямоугольники - среднее время, затрачиваемое на один шаг из рис. 2 ; кружки - зависимость средней длины шага, равная произведению средней скорости (рис. 1) на среднее время, затрачиваемое на один шаг, от состояния пациента.

аритмии походки до тап-теста. После тап-теста походка становится более ритмичной. Походка продолжает оставаться ритмичной и после установки шунта.

На рис. 3, $a$ изображены зависимости амплитуды размаха мгновенных скоростей для пациента С. и здорового человека, там же приведена средняя скорость движения на марше. Видно, что по мере выполнения медицинских манипуляций оба значения скоростей возрастают, отличаясь, однако, от соответствующих показателей здорового человека.

На рис. 3, $b$ показана зависимость времени, затрачиваемом на шаг, и средней длины шага (произведение средней скорости на время шага) от состояния пациента. Видно, что время, затрачиваемое на шаг, уменьшается после тап-теста и установки шунта, а длина среднего шага возрастает. Эти параметры приближаются к параметрам здорового человека.

Итак, анализ данных, представленных на рисунках, свидетельствует об улучшении клинически-значимых параметров походки пациента после тап-теста и операции вентрикулоперитонеального шунтирования, поскольку эти параметры имеют тенденцию к стремлению к параметрам, характерным для здорового человека.

Также, отмеченное улучшение походки после тап-теста, диагностировавшееся по методу, изложенному выше, сопровождалось у пациента С. регрессом когнитивных нарушений, что подтверждено проведением нейропсихологических тестов. Эти обстоятельства позволили установить диагноз: нормотензивная гидроцефалия Хаким-Адамса в стадии субкомпенсации.

С учетом имеющихся данных клинической картины MPT головного мозга, установленного диагноза больному выполнено оперативное вмешательство - вен- трикулоперитонеальное шунтирование с применением внешнерегулируемого клапана с антисифоном. В послеоперационном периоде при оценке результатов операции отмечена положительная динамика в виде улучшения походки (что доказано с помощью разработанного прибора), а также снижения когнитивных нарушений (по результатам нейропсихологического тестирования). Таким образом, отмечено типичное течение послеоперационного периода после адекватной коррекции нормотензивной гидроцефалии, что ex juvantibus подтвердило диагноз основного заболевания.

Более того, как оказалось, что именно средняя скорость движения пациента по дистанции и время, затрачиваемое пациентом на один шаг, чувствительны к состоянию пациента до операции, при тап-тесте и после ликворошунтирующей операции.

\section{Вывод}

Представленный прибор и разработанная методика анализа полученных с его помощью грессограмм и целеритограмм, являются эффективными для оценки походки у пациентов с нормотензивной гидроцефалией и могут оказаться перспективными для создания аппаратно-программного комплекса на основе ультразвукового дальномера и элементарных программ обработки телеметрической информации. Такой комплекс является перспективным для широкого применения в практике работы врачей-неврологов и нейрохирургов. 


\section{Список литературы}

[1] Levine D.N. // Bull. Math. Biol. 1999. Vol. 61. N 5. P. 875-916.

[2] Adams R.D., Fisher C.M., Hakim S., Ojemann R.G., Sweet W.H. // New England J. Medicine. 1965. Vol. 273. N 3. P. 117-126.

[3] Virhammar J., Cesarini K.G., Laurell K. // Eur. J. Neurol. 2012. Vol. 19. N 2. P. 271-276.

[4] Agostini V., Lanotte M., Carlone M., Campagnoli M., Azzolin I., Scarafia R., Massazza G., Knaflitz M. // Arch. Phys. Med. Rehabil. 2015. Vol. 96. N 7. P. 1235-1241.

[5] Ishikawa M., Yamada Sh.K. // Fluids Barriers CNS. 2016. Vol. 13. N 20. P. 1-7.

[6] Bassar B.R., Lippa C. // Gerontology and Geriatric Medicine. 2016. Vol. 2. P. 1-6.

[7] Shinoda N., Hirai O., Hori S., Mikami K., Bando T., Shimo D., Kuroyama T., Kuramoto Y., Matsumoto M., Ueno Y. // J. Neurosurg. 2017. Vol. 3. P. 1-7.

[8] Cagnin A., Simioni M., Tagliapietra M., Citton V., Pompanin S., Della Puppa A., Ermani M., Manara R.A. // J. Alzheimer. Dis. 2015. Vol. 46. N 4. P. 1033-1038.

[9] D'Costa D.F., Sheehan L.J., Phillips P.A., Moore-Smith B. // Age Ageing. 1995. Vol. 24. N 3. P. 210-212.

[10] Stolze H., Kuhtz-Buschbeck J.P., Drücke H., Jöhnk K., Illert M., Deuschl G. // J. Neurol Neurosurg Psychiatry. 2001. Vol. 70. P. 289-297. 